Mantovan L. \& C. Geraci (2018). R-impersonal interpretation in Italian Sign

Language (LIS). In G. Barberà \& P. Cabredo Hofherr (eds.), Reference Impersonals

in Sign Languages, special issue of Sign Language \& Linguistics 21 (2), 232-257

\title{
R-impersonal interpretation in Italian Sign Language (LIS)
}

Lara Mantovan ${ }^{1} \&$ Carlo Geraci ${ }^{2}$

issn 1387-9316 I

e-issn 1569-996x

1

University of Milan-Bicocca; Ca' Foscari University Venice

2

CNRS, Institut Jean-Nicod; ENS, Département d'Etudes Cognitives, Paris

\section{Please note that this article is under copyright and the publisher should be contacted for permission to re-use or reprint the material in any form.}

In this paper, we examine agent backgrounding in Italian Sign Language (LIS). Specifically, we are interested in identifying and describing the strategies used by LIS signers to reduce referentiality. On the basis of low-referential contexts (cf. questionnaire in the Introduction chapter), we recorded target sentences containing potential markers of agent backgrounding and asked three LIS native signers to provide felicity judgments on them using a 7-point scale. We discuss agent-backgrounding strategies of different types: i) manual, ii) nonmanual, and iii) syntactic. Overall, our study shows that the combination of raised eyebrows and mouth-corners down associated with the existential quantifier SOMEONE and the sign PERSON makes the agent-backgrounding reading more prominent. Other strategies that can be used in LIS to reduce referentiality are free relatives, perspective shift, and null subject. We also investigate in more detail the semantic status of SOMEONE, PERSON, and the null subject through well-established tests from the literature.

Keywords: Italian Sign Language, agent backgrounding, referentiality, impersonals, indefinite pronouns

\section{Introduction}

In this paper, we present some preliminary findings on how R(eference)-impersonals (Siewierska 2011) are conveyed in Italian Sign Language (LIS). Although a growing number of studies have been investigating various aspects of the grammar of this language, the issue of agent backgrounding (for a detailed description of this linguistic phenomenon, see the Introduction chapter) represents a severely understudied topic. In an attempt to overcome this gap, the present study aims at identifying and describing the strategies employed by LIS signers to reduce referentiality. Our starting hypotheses were that agent backgrounding is triggered by: i) manual strategies (pointing pronouns, lexical signs such as SOMEONE and PERSON, etc.), ii) non-manual strategies, and iii) a combination of manual and non-manual strategies.

On the basis of new data collected through fieldwork, we will show that a combination of lexical items (i.e. the signs SOMEONE and PERSON) and non-manual components (i.e. raised eyebrows, raised chin, and lowered mouth corners, here labeled as 'ab', for agent backgrounding) as well as null subjects can trigger agent backgrounding in LIS. A preview of 
these strategies is given in (1). ${ }^{1}$

(1) a. SOMEONE HOUSE POSS 1 ENTER
b. PERSON HOUSE POSS 1 ENTER

'Someone entered my house.'

c. pro HOUSE POSS 1 ENTER

'They entered my house.'

Other strategies in addition to those illustrated in (1) are investigated such as free relatives and perspective shift. As a point of comparison, we also consider morphological strategies such as the use of high loci, which have been shown to be crucial in conveying non-specificity and domain widening in Catalan Sign Language (LSC) and American Sign Language (ASL) (see Barberà \& Cabredo Hofherr 2017a; Davidson \& Gagne, in prep.). In the second part of the paper, we will focus on the semantic properties of the constructions in (1) and we will show that the signs SOMEONE and PERSON accompanied by ' $a b$ ' facial expression behave as indefinite pronouns, while the null subject strategy is a good candidate to convey impersonal readings.

The paper is structured as follows. In section 2, we present a short overview of basic information about LIS. Section 3 illustrates how data collection was conducted. Section 4 focuses on the agent-backgrounding devices used in LIS. Section 5 demonstrates through empirical evidence that SOMEONE and PERSON function as indefinite pronouns and the null subject functions as an impersonal pronoun. Finally, section 6 concludes the paper.

\section{Background information about LIS}

LIS is the sign language used by the signing Deaf community in Italy and Ticino, the southernmost canton of Switzerland. In this area, there are approximately 70,000 people who were born deaf or became deaf before acquiring spoken Italian. Of these, Eugeni (2008) estimates that about $60 \%$, that is about 42,000 deaf people, had access to LIS as their first language, although the language is not officially recognized by the Italian Government yet.

In this section, we provide a quick overview of some properties of LIS that will be relevant to the rest of the paper. Although word order in simple declarative sentences appears quite flexible (Branchini \& Geraci 2011), our informants have a strong preference for S(ubject)- O(bject)-V(erb) order, as illustrated in (2) (see Cecchetto, Geraci \& Zucchi 2006 for a more comprehensive overview of the syntactic properties of LIS). Other orders are also possible, especially when a constituent is topicalized or focalized. Specifically, topicalized

\footnotetext{
${ }^{1}$ Abbreviations in the examples: $a b=$ agent backgrounding, $b r=$ brow raise, cond $=$ conditional, def $=$ definite, indef $=$ indefinite, rel $=$ relativization, top $=$ topicalization, $u p=$ articulation in a high location in signing space.
} 
constituents are marked by a specific prosodic contour realized by brow raise (here marked as 'br') and appear at the beginning of the sentence. A couple of examples are provided in (3a) and (3b), which show OSV and SVO patterns, respectively (Geraci, Cecchetto \& Zucchi 2008:47). The non-manual markers (NMMs) are indicated by a line on top of the sign glosses they co-occur with.

(2) GIANNI COFFEE ORDER

'Gianni ordered a coffee.'

top (br)

(3) a. COFFEE GIANNI ORDER

top (br)

b. GIANNI ORDER COFFEE

'Gianni ordered a coffee.'

The distribution of NMMs determines specific constructions. For example, if -clauses in LIS are not introduced by an overt lexical sign corresponding to the English connective if. Rather, they are normally marked by raised eyebrows co-occurring with the antecedent of the conditional clause (Barattieri 2006).

(4) GIANNI ANSWER WRONG, IX 3 ELIMINATE

'If Gianni gives the wrong answer, he will be eliminated.'

The role of NMMs is crucial not only to the syntactic structure of LIS. A number of recent studies have shown that NMMs are systematically used to express some constructions with specific semantic properties and convey nuances in meaning (Zucchi 2004, 2009; Mantovan \& Geraci 2015, a.o.). Working on the syntax of cardinal numerals, Mantovan \& Geraci (2015) show that if a cardinal is included in an indefinite nominal expression, i.e. a Determiner Phrase (DP) associated with a new referent in the discourse, it usually co-occurs with backward-tilted head and raised eyebrows (here labeled as 'indef'). If the cardinal is included in a definite nominal expression, i.e. a DP associated with an already-mentioned referent, it usually co- occurs with squinted eyes, lowered eyebrows, and chin down (here labeled as 'def'). These two distinct sets of NMMs are illustrated in the images below.

--- Insert Figure 1 and Figure 2 ---

The relevant examples are provided in (5). The glosses show that: i) the relative order of the cardinal with respect to the noun is not enough to distinguish between definiteness and indefiniteness and ii) NMMs play a crucial role in determining the correct interpretation.

(5) a. CHILD TWO / TWO CHILD ... 


\section{'Two children' \\ def}

b. CHILD TWO ...

'The two children'

In the next sections, we will capitalize on these facts to investigate agent backgrounding in LIS. Specifically, the use of the indefinite facial expression will be shown to co-occur with some additional features to convey reduced referentiality.

\section{A note on the methodology}

We collected the linguistic data for this study through fieldwork (interaction with nativesigning informants). In order to keep as much distance as possible from spoken and written Italian, we adopted the procedure illustrated in the Introduction of this issue with some minor changes. All the data were collected following the 'playback method' (Schlenker 2011), which essentially involves two steps: i) data elicitation (see section 3.1) and ii) data assessment through a sentence matching task (see section 3.2).

Linguistic judgments were provided by three LIS native signers, two men and one woman. At the time of data assessment, they were aged between 35 and 57. Two of them come from Southern Italy and one comes from Northern Italy. They are all Deaf, have Deaf parents, and have been exposed to LIS since birth. As for their education, one of them earned a Master degree while two hold a high school degree. ${ }^{2}$

\subsection{Data elicitation}

Elicitation is composed of two parts: i) creation of the relevant scenarios and ii) elicitation of the target sentences.

As for the scenarios, we referred to the contexts included in the questionnaire on impersonal reference (see Introduction chapter) and selected one scenario for each semantic environment (e.g. one scenario for anchored existential, one for vague existential, and so on). The authors of this paper, a professional interpreter and a native signer of LIS, translated and recorded the scenarios in LIS. None of the manual and non-manual items under investigation in this paper were used to create the scenarios.

The second part of data elicitation was aimed at obtaining a paradigm of target sentences for each scenario. The target sentences are simple sentences differing only in one sign, namely the potential marker of agent backgrounding. Specifically, we considered twelve strategies: pointing pronouns $\left(\mathrm{IX}_{1}, \mathrm{IX}_{2}, \mathrm{IX}_{3}, \mathrm{IX}_{3 \mathrm{p}}\right)$, a lexical variant of the third-person pronoun

2 The elicited data were produced by two Deaf actors (Mirko Santoro and Lorenzo Laudo). Felicity judgments in the sentence matching sessions were provided by three native-signing informants (Mirko Santoro, Valerio Andrioli, and Vincenza Giuranna). We thank them all for their contribution and we also thank Gabriele Caia, who helped us in the preliminary stage of data collection. 
instantiated by a B-handshape (here labeled as B-PRONOUN), ${ }^{3}$ the singular and plural version of the sign for person (PERSON, PERSON $\left.{ }_{\mathrm{pl}}\right),{ }^{4}$ the existential quantifier SOMEONE, ${ }^{5}$ the sign for ONE, full NPs, the null subject, and the wh-sign WHO embedded in free relatives. We also tested the phrasal combination ONE PERSON (for details on the use of the indefinite determiner in LIS, see Mantovan 2017) and the signs HEARING ${ }^{6}$ and DEAF. We also tested morphophonological manipulations of duration and loci. Specifically, we tested: i) $\mathrm{IX}_{3}$ and $\mathrm{IX}_{3 \mathrm{pl}}$ when realized as strong vs. weak pronouns, and ii) the third-person pronouns, PERSON, ONE PERSON, SOMEONE, ONE realized in standard and high loci. The difference between strong and weak pointing signs lies both in movement duration and palm orientation: the former are realized with a longer and more intense movement and palm down, whereas the latter are realized with a short trajectory and palm up (for more details see Bertone \& Cardinaletti 2011). These morphophonological manipulations will be further discussed in section 4.1.2.

The complete list of target sentences we elicited is presented in (6). For illustrative purposes, we exemplify the procedure by the example of the vague existential context.

(6) Scenario: You arrive at home after a long vacation and you find the door of your apartment broken.

Initial pool of target sentences (illustrative examples):

a. IX HOUSE POSS $_{1}$ ENTER

b. $\mathrm{IX}_{2}$ HOUSE POSS ${ }_{1}$ ENTER

c. $\mathrm{IX}_{3[ \pm] \text { up-weak }}$ HOUSE POSS ${ }_{1}$ ENTER

d. $\mathrm{IX}_{3[ \pm] \text { up-strong }}$ HOUSE POSS ${ }_{1}$ ENTER

e. $I_{3 \mathrm{pl}[ \pm] \text { up-weak }}$ HOUSE POSS $_{1}$ ENTER

f. $\mathrm{IX}_{3 \mathrm{pl}[ \pm] \text { up-strong }}$ HOUSE POSS $_{1}$ ENTER

g. PERSON ${ }_{[ \pm] \text {up }}$ HOUSE POSS $_{1}$ ENTER

h. ONE PERSON ${ }_{[ \pm] \text {up }}$ HOUSE POSS ${ }_{1}$ ENTER

i. PERSON ${ }_{\mathrm{pl}}$ HOUSE POSS $_{1}$ ENTER

j. SOMEONE $_{[ \pm] \text {up }}$ HOUSE POSS ${ }_{1}$ ENTER

k. ONE ${ }_{[ \pm] \text {up }}$ HOUSE POSS $_{1}$ ENTER

1. WHO HOUSE POSS ${ }_{1}$ ENTER

m. VISITOR HOUSE POSS ${ }_{1}$ ENTER

n. pro HOUSE POSS ${ }_{1}$ ENTER

o. B-PRONOUN HOUSE POSS ENTER $_{1}$

${ }^{3}$ This pronoun is realized as a $\mathbf{B}$-handshape oriented toward a locus in the signing space with a vertical (topdown) single path movement.

${ }^{4}$ The sign PERSON is realized by a little C-handshape combined with a vertical top-down single movement.

${ }^{5}$ Similarly to other sign languages (e.g. French Sign Language), this sign is realized with an index handshape pointing upwards, the hand and the arm are active articulators while the movement consists in a repeated rotation operated at the elbow node.

${ }^{6}$ It has been observed that, at least in some cases, the sign HEARING in LIS may convey generic reference and exclusivity in scenarios with a negative connotation (Geraci 2012). 


\section{p. HEARING HOUSE POSS 1 ENTER \\ q. DEAF HOUSE POSS 1 ENTER}

In order to test the role of the manual and non-manual components separately, we elicited the whole set of target sentences twice. In one session, we asked the signers to produce the sentences with the facial expressions typical of standard declarative sentences (i.e. nonmarked NMMs). These are illustrated in Figure 3.

\section{--- Insert Figure 3 ---}

In the other session, they were asked to produce the same sentences with specific NMMs functioning as potential markers of agent backgrounding (for an overview of the syntactic and semantic functions of NMMs, see section 2). These features, which have been identified on the basis of the informants' feedback, are raised eyebrows, chin slightly raised, and mouth corners pulled downward (see Figure 4). These videos were then used to assess acceptability and felicity judgments against the various scenarios.

\section{--- Insert Figure 4 ---}

In the next sections, these NMMs will emerge as crucial for the expression of agent backgrounding in LIS.

\subsection{Context-sentence matching}

Once the whole paradigm of target sentences was ready, we proposed a sentence matching task to all three informants. With each of them, we collected felicity judgments for eleven contexts. The procedure was as follows: i) the informant watched the scenario; ii) $s /$ he watched a randomly picked target sentence; iii) $\mathrm{s} /$ he evaluated to what extent the sentence is felicitous in the given scenario.

Felicity judgments were collected using a 7-point scale ( 1 being totally infelicitous and 7 perfectly felicitous). For each sentence, we asked the informants to express two judgments: one for the agent-backgrounding reading and one for the referential reading. We repeated steps ii) and iii) for all target sentences, both with the facial expression shown in Figure 3 and the one shown in Figure 4. Unavailable semantic interpretations are marked by the hash sign (\#) throughout the paper. The sentences marked by the \# symbol have been judged on average equal to or less than 2 by our informants.

This initial stage helped us detect a set of likely candidates for agent backgrounding. Later on, a more refined fieldwork with two informants was conducted to collect qualitative evidence.

\section{Agent-backgrounding strategies}


The main strategies that our informants used to convey agent backgrounding are presented in this section: first we discuss the manual strategies, second we focus on the non-manual components, third we illustrate the syntactic strategies.

For the sake of brevity, we use examples from the vague existential scenario to illustrate the data, although we tested all scenarios reported in the Introduction of this issue. The scenario and the baseline sentence are given in (7).

(7) Scenario: You and your partner come back home after a long vacation. Once next to the door of your house you realize that it is open.

Baseline sentence: THIEF HOUSE POSS 1 ENTER

'A thief entered my house.'

Full NPs encoding indefinite/non-specific/generic subjects are always acceptable, indicating that some kind of agent backgrounding can be encoded in the language even if the language does not have overt indefinite determiners (Mantovan \& Geraci 2015).

\subsection{Manual strategies}

The data reported in this section refer to manual markers used in standard sentences without the systematic presence of any non-manual component that could convey agent backgrounding. We first investigate the lexical options without co-occurring NMMs because we want to tease apart the contribution of manual signs from that of the non-manual components, and see how general these strategies are with respect to the various contexts illustrated in the Introduction chapter. Thus, the data presented in this section always have the prosodic contour used in standard declarative sentences.

The manual markers investigated in this study are of two types: 1) free morphemes, i.e. independent lexical signs and 2) manual bound morphemes, i.e. morphemes affecting some formational parameters of the sign they affix to. ${ }^{7}$ As for lexical signs, we evaluated the existential quantifier SOMEONE, possible pronominal uses of the sign ONE, and the sign PERSON. For these signs, we had preliminary evidence that they could be used in agentbackgrounding contexts. As for bound morphemes, we manipulated sign location to study the contrast between standard vs. high loci (as, according to Barberà 2015, this feature has been shown to be relevant in LSC) and the speed/duration of pointing signs to contrast weak vs. strong versions of pointing pronouns (as, according to Bertone \& Cardinaletti 2011, this feature has been shown to trigger interpretational differences in LIS).

\footnotetext{
${ }^{7}$ Lexical NMMs aside, we assume that NMMs act at the interface between morphology and prosody since spreading may co-occur with more than one lexical item, even if the "prosodic morpheme" is attached to a single stem. We remain neutral as to whether this aspect of prosodic morphology is driven by a null morpheme or is syntactically driven by the interaction between features on the nominal expression and the functional structure of the clause.
} 


\subsubsection{Lexical markers}

The major observation is that without ' $a b$ ' NMMs, agent-backgrounding readings are extremely hard to obtain. The general picture in this respect is that the referential (specific) ${ }^{8}$ reading is always preferred and, in most cases, it seems to be the only reading available. This is certainly true for first- and second-person pronouns, but also for third-person pronouns, both singular and plural, ${ }^{9}$ as shown in (8). The examples produced here referred to the context given in (7). Crucially, they are all grammatical: the contrast is found in the intended interpretations. This is the reason why diacritics are placed on the English translations rather than on the LIS examples.
(8)
a. IX 1 HOUSE POSS 1 ENTER
\# 'I entered my house.' (pragmatically odd)
\# 'Someone entered my house.' (agent-backgrounding reading not available)
b. IX HOUSE POSS $_{1}$ ENTER
'You entered my house.' (referential reading only)
\# 'Someone entered my house.' (agent-backgrounding reading not available)
c. IX I HOUSE POSS $_{1}$ ENTER
'S/he entered my house.' (referential reading only)
\# 'Someone entered my house.' (agent-backgrounding reading not available)
d. IX $_{3 \mathrm{pl}}$ HOUSE POSS ${ }_{1}$ ENTER
'They entered my house.' (referential reading only)
\# 'Someone entered my house.' (agent-backgrounding reading not available)

The use of a first-person pronoun results in extremely awkward sentences since the scenarios normally exclude the signer as the agent of the event described in the target sentence. Secondperson pronouns are acceptable as long as the signer actually knows or is able to identify the addressee as a participant in the event, e.g. in the case of the scenario in (7), the signer must know that the addressee broke into the apartment. For third-person pronouns, the sentences are acceptable as long as the signer knows who the relevant individual(s) are who broke into the house. Crucially, agent-backgrounding readings are unavailable with pointing pronouns.

In addition to standard pointing pronouns encoding first, second, and third person, we tested another pronoun, namely the B-PRONOUN (see footnote 2). Also in this case, the agentbackgrounding reading is unavailable, as shown in (9). ${ }^{10}$

B-PRONOUN HOUSE POSS ${ }_{1}$ ENTER

'He entered my house.' (referential reading)

\# 'Someone entered my house.' (agent-backgrounding reading not available)

\footnotetext{
${ }^{8}$ In this paper, specificity is intended in terms of identifiability (see von Heusinger 2002).

${ }^{9}$ The third-person pronouns produced by our signers point toward an arbitrary ipsilateral locus. The plural form is realized with the index finger making a small circle in neutral space.

${ }^{10} \mathrm{We}$ tested for a third-person singular reference. However, if oriented appropriately, the pronoun can be used to refer to second and first person.
} 
These results seem to indicate that directionality both in the form of a directional movement or orientation toward a locus is a strong cue to resolve pronominal reference by picking some individual who is contextually salient. The issue is then to determine the morphological exponent that is responsible for that reading. A possible candidate are the locations in space towards which pointing signs and orientation are directed. The role of space has been proven to crucially affect referentiality both in LSC and ASL (Barberà 2015; Davidson \& Gagne, in prep.), so it is likely to play a non-trivial role in LIS as well. One possibility is that these locations in space strongly bias personal reference, hence in the previous examples, loci are the morphological exponents of specific/definite reference. Notice that our target sentences do not force real space uses (i.e. do not necessarily create connections with the physical environment including the signer's body and its surroundings). However, considering that all target sentences come after a scenario that creates the relevant context, abstract space is also quite hard to obtain. The most likely situations, which we assume to be the default in our data, are those in which blended space is used (Liddell 2003). ${ }^{11}$

That the spatial component is relevant in resolving pronominal reference is confirmed by the fact that agent-backgrounding readings are unavailable even with PERSON. This sign does not contain directional features, with the only exception that its hand-orientation is reversed when it is used to refer to first person (as it occurs with the B-PRONOUN). Still, its location in the signing space as determined by the top-down vertical movement seems to force a definite/specific reading, as indicated in (10a).

a. PERSON HOUSE POSS ${ }_{1}$ ENTER

'That person entered my house.' (referential reading)

\# 'Someone entered my house.' (agent-backgrounding reading not available)

b. PERSON $\mathrm{pl}_{\mathrm{pl}}$ HOUSE POSS 1 ENTER

'These people entered my house.' (referential reading)

\# 'They entered my house.' (agent-backgrounding reading not available)

The same result is found when the reduplicated plural version is used, as in (10b). Interestingly in the case of the plural version of the sign PERSON, the indefinite/impersonal reading is available in the unrestricted universal context, as shown below.

(11) Scenario: You read about an expedition that is preparing to cross a desert. You point out to your child that the members should prepare carefully and take plenty of water. Your child asks for clarifications.

SURVIVE, PERSON ${ }_{\mathrm{pl}}$ WATER NEED

'People need water to survive.' (agent-backgrounding reading)

\footnotetext{
${ }^{11}$ Simplifying a bit, signing space is used as blended space any time a mental image concerning space is mapped onto signing space.
} 
The possibility for a plural/collective NP to trigger a generic interpretation is not surprising since it is found in other languages as well. For example, in French les gens ("the people") has a generic reading in the unrestricted universal context (les gens ont besoin d'eau pour survivre), but not in the vague existential context (les gens sont entrés dans ma maison).

Differently from the lexical markers discussed up to this point, where agentbackgrounding readings are generally not available, in the case of SOMEONE, we have been able to obtain such readings, as shown in (12). Like their English equivalent, target sentences with SOMEONE are normally ambiguous between a specific and a non-specific reading. Still, in these cases, the most accessible reading is the specific one. As we illustrate in the next section, the presence of a particular set of NMMs reverses this bias.

\section{SOMEONE HOUSE POSS ${ }_{1}$ ENTER}

'Someone entered my house.' (both referential and agent-backgrounding readings)

Finally, we investigated if the sign ONE alone could be used as a pronominal element with agent backgrounding. ${ }^{12}$ While it is clear that LIS does not lexically mark the distinction between definite and indefinite articles in pointing signs, Mantovan \& Geraci (2015) report in a corpus-based study on nominal modification that some signers of LIS use the sign ONE as an indefinite article. Therefore, we checked whether this sign could be used as an indefinite pronoun. Unfortunately, none of our informants accept the pronominal use of ONE, therefore we could not test this item. ${ }^{13}$

Of all the pronominal forms and quantifiers we considered in this section, only the sign SOMEONE is able to systematically convey agent backgrounding in the absence of cooccurring NMMs.

\subsubsection{Manual morphemic markers}

Building on the seminal work by Barberà (2015) and Barberà \& Cabredo Hofherr (2017a) on LSC, where the alternation of high vs. standard loci encodes a contrast in specificity and affects the availability of indefinite readings of pronouns and quantifiers, we investigated whether a similar contrast in space gives analogous results in LIS. Specifically, we manipulated standard vs. high loci of third-person pronouns (singular and plural), the signs PERSON, PERSON ${ }_{\mathrm{pl}}$, ONE, and SOMEONE. For the sake of brevity, we report here only examples with SOMEONE, however, the effect of high loci is the same with the other manual signs. The example (12) repeated here as (13a), contains the sign SOMEONE articulated in a standard location in space.
a. SOMEONE HOUSE POSS ENTER $_{1}$
b. SOMEONE ${ }_{\text {up }}$ HOUSE POSS ${ }_{1}$ ENTER

12 The pronominal use of ONE was excluded by previous work (Mantovan 2017).

${ }^{13}$ The use of ONE as an indefinite article was stronger in the older population of signers (Mantovan \& Geraci 2015). Our signers are relatively young, so it is not unexpected that they cannot use ONE as an indefinite marker. However, the extended index handshape can be used in LIS as a whole-entity classifier for person. 
'Someone (whom I know and who is located higher, e.g. lives upstairs) entered my house.' (referential reading)

The use of high loci induces an even stronger bias toward a referential reading, as indicated by the English translation. Furthermore, agent-backgrounding readings become harder to get.

Differently from what has been reported for LSC, LIS does not seem to encode nonspecific readings by simply manipulating the location of signs with respect to $[ \pm$ high] feature. We also controlled for the direction of eye gaze, as we know that interrupting eye contact with the addressee normally increases definiteness as a blending with real space might be involved. In the example in (13b), the gaze of the signer is towards the addressee. Although not systematically for all contexts, we checked the effect of eye gaze towards high loci and we did not find any substantial difference. It should also be noted that high loci in sign languages may introduce a variety of nuances such as making explicit a precise location in a scene or giving honorific status to the referent (e.g. in the case of nouns referring to institutional titles such as PRESIDENT). It is then possible that differently from other sign languages, high loci in LIS convey indefiniteness or non-specific interpretations only as a very weak inference (like easily defeasible implicatures) and this is the reason why we were not able to detect any effect. We leave this issue for future research.

The other parameter we manipulated was the movement component of pointing signs. Bertone \& Cardinaletti (2011) report that pointing pronouns that have a short internal movement can be used as expletives in weather constructions as in (14).

IX $\mathrm{X}_{\text {up-weak }}$ RAIN

'It rains.'

We checked whether these pronouns can also convey agent backgrounding. The results we obtained is that standard vs. weak pointing signs do not differ, in that neither is used to express reduced referentiality. The example in (8c) is repeated below as (15a).

a. IX 3 HOUSE POSS 1 ENTER

b. IX $_{3 \text {-weak }}$ HOUSE POSS 1 ENTER

'S/he entered my house.' (referential reading)

\# 'Someone entered my house.' (agent-backgrounding reading not available)

To sum up, none of the morphological strategies that we tested seems to convey agent backgrounding in LIS. The fact that the contrast between standard vs. high loci does not seem to produce the same strong effects documented for LSC opens interesting typological perspectives in terms of cross-linguistic variation that we leave for future research. The fact that weak pointing signs can serve as expletives has no apparent extensions or consequences in other domains of pronominal reference. ${ }^{14}$ Finally, SOMEONE can be used to convey

14 The fact that weak pronouns have not been documented in other environments where expletives are normally 
referential reduction, possibly indicating that this quantifier is likely to become an independent marker of agent backgrounding or even that it is genuinely ambiguous/underspecified.

\subsection{Non-manual strategies}

In this section, we report the effect of NMMs in conveying agent backgrounding. Rather than testing several combinations of non-manual features, we focused on the ones that after a preliminary investigation seemed to be most promising. Specifically, we systematically investigated the combination of raised eyebrows and mouth corners down (see Figure 4 in section 3.1). In the target sentences we tested, these NMMs co-occur with the lexical signs investigated in section 4.1 and do not spread over larger portions of the sentence. This was the most natural way to use that combination of NMMs for our actors/informants. The overall effect of the presence of this combination of NMMs is twofold: on the one hand, it reduces felicity and acceptability of the referential reading (with some exceptions, see below); on the other hand, it selectively increases access to agent-backgrounding readings. The strength of the effect is not uniform. Indeed, in some scenarios we observed small differences with respect to the judgments provided for the target sentences without this set of NMMs, in other cases the effect is larger.

In the case of pointing signs (either weak or strong, singular or plural) the presence of this particular set of NMMs does not seem to make agent-backgrounding readings available. However, it slightly reduces acceptability of the referential readings. In addition to that, the NMMs introduce a nuance of uncertainty. The signer is not sure whether the referent actually participated in the event described in the target sentence, as indicated by the English translation of the examples in (16). ${ }^{15}$ Alternatively these NMMs are interpreted as indicating that the signer does not know the identity of the referent. The degrading effect in terms of acceptability is stronger with strong pronouns than with weak pronouns.

$\underline{\mathrm{ab}}$

(16) a. ?? IX $\mathrm{IX}_{3}$ HOUSE POSS 1 ENTER

$\mathrm{ab}$

b. ? IX ${ }^{-}$-weak HOUSE POSS 1 ENTER

'S/he might have entered my house, but I am not sure.' (referential reading)

'S/he might have entered my house, but I do not know the identity of that person.' (referential reading)

\# 'Someone entered my house.' (agent-backgrounding reading not available)

$\mathrm{ab}$

found, like in raising and tough-constructions (where null expletives are regularly found, see Geraci 2014) may indicate that the upward movement makes the pointing sign found in weather constructions somehow special.

${ }^{15}$ We suspect that in these contexts, the NMMs are introducing an inference of some sort. Further research is needed to establish the nature of the inference, whether it is an implicature, a presupposition of the standard type, or a co-supposition (Schlenker 2016). 
c. * ${ }^{2} \mathrm{X}_{3 \mathrm{pl}}$ HOUSE POSS $_{1}$ ENTER

$\mathrm{ab}$

d. ? IX I $_{\text {3pl-weak }}$ HOUSE POSS 1 ENTER

'They might have entered my house, but I am not sure.' (referential reading)

\# 'They entered my house.' (agent-backgrounding reading not available)

The strongest effect of the NMMs is found in combination with the sign for SOMEONE and PERSON. In these cases, the referential reading becomes completely unavailable and only the agent-backgrounding one is available.

$\mathrm{ab}$

(17) SOMEONE/PERSON HOUSE POSS ENTER $_{1}$

\# 'Someone that I know entered my house.' (referential reading not available)

'Someone entered my house.' (it could be anyone, agent-backgrounding reading)

Finally, we checked the combined effect of high loci and NMMs. The idea was to see whether the $[ \pm$ high $]$ loci pattern may emerge in LIS as a combined effect of loci manipulation and NMMs.

In the case of pointing pronouns (we systematically checked weak third-person singular pronouns), impersonal readings do not emerge and referential uses are degraded in a similar way as in pointing pronouns directed towards the standard height. The example in (9) is repeated below as (19a).

(18) a. ? IX IX-weak HOUSE POSS $_{1}$ ENTER

$\mathrm{ab}$

b. ? $\mathrm{IX}_{3 \text {-up-weak }}$ HOUSE POSS ${ }_{1}$ ENTER

'S/he might have entered my house, but I am not sure.' (referential reading)

\# 'Someone entered my house.' (agent-backgrounding reading not available)

(19) a. B-PRONOUN HOUSE POSS 1 ENTER

'He entered my house.' (referential reading)

\# 'Someone entered my house.' (agent-backgrounding reading not available)

$\mathrm{ab}$

b. * B-PRONOUN HOUSE POSS 1 ENTER

As for SOMEONE, there seems to be no effect when the sign is produced in a location higher in space than when produced in its standard place of articulation. Still, access to the referential reading is problematic. The example in (17) is repeated below as (20a).

(20) a. SOMEONE HOUSE POSS ENTER $_{1}$

$\mathrm{ab}$ 
b. SOMEONE up HOUSE POSS $_{1}$ ENTER

\# 'Someone whom I know entered my house.' (referential reading not available)

'Someone entered my house.' (it could be anyone, agent-backgrounding reading)

To sum up, the combination of raised eyebrows and mouth corners down can be interpreted as a generalized marker of uncertainty. When combined with pointing pronouns or NPs, this combination of NMMs is interpreted as the signer not being sure that the individual denoted by the pronoun or the noun phrase is actually involved in the event. In the case of SOMEONE and PERSON, it makes the referential reading unavailable and the agent-backgrounding reading more prominent.

\subsection{Syntactic strategies}

We illustrate here the syntactic strategies that are potential candidates for conveying agent backgrounding. These are the use of null subjects, free relatives, and constructions involving perspective shift.

Agent-backgrounding readings are available with null subjects, as shown in (21). Although there is no overt plural marker on the verb, the sentence is compatible with a plural referent. As with SOMEONE, the target sentences are ambiguous between referential and impersonal readings in this case, as well. However, the informants do not show a specific bias towards one or the other.

\section{pro HOUSE POSS ${ }_{1}$ ENTER}

'They/someone entered my house.' (both referential and agent-backgrounding readings)

Combining the use of agent-backgrounding NMMs with the null subjects has proven quite difficult since the NMMs should be associated with some lexical material corresponding to the pronominal element. Spreading on either the verb or over the entire clause produces the effect of uncertainty already discussed above. The data in (22) incidentally confirm that the most natural distribution for the NMMs associated with agent backgrounding is on DPs.

(22) a. pro HOUSE POSS 1 ENTER

$\mathrm{ab}$

b. pro HOUSE POSS 1 ENTER

'They/someone might have entered my house.' (agent-backgrounding reading)

Another option to convey agent backgrounding is by means of free relatives (e.g. whoever did that was irresponsible). To construct standard relative clauses (Cecchetto, Geraci \& Zucchi 2006), LIS signers use a determiner-like pronoun, which is glossed as PE after Branchini \& Donati (2009), and special NMMs (here labeled as 'rel' and consisting of raised eyebrows, a specific tension of the eyes and upper cheeks, and a slight forward head tilt). These features 
are represented in (23a). The sign PE can also be used as a resumptive pronoun for sentential complements (Geraci, Cecchetto \& Zucchi 2008; Geraci \& Aristodemo 2016), as shown in (23b).

- . . . . _ rel

(23) a. BOY CALL PE LEAVE DONE

'The boy that called left.'

br

b. PIERO CAR STEAL MARIA PE TELL

'Maria said that Piero stole a car.'

Curiously, the determiner-like pronoun PE cannot be used in free relatives, which are only possible once the relevant wh-sign is used, as shown in (24) from Branchini (2009: 104).
a. * EXAM DO PE LEAVE CAN ${ }^{16}$
rel
b. EXAM DO WHO LEAVE CAN

'Whoever completed the exam can leave.'

This is also confirmed by our informants. Once tested in the relevant scenarios, PE-relatives cannot be interpreted as free relatives and always get a fully referential reading; while free relatives with wh-pronouns can convey agent backgrounding, as shown in (25).

rel

(25) a. PERSON ENTER PE SURE WANT STEAL

'The person who entered (and I know who s/he is) wanted to steal something.' (referential reading)

rel

b. ENTER WHO SURE WANT STEAL

'Whoever entered wanted to steal something.' (agent-backgrounding reading)

The last strategy we discuss is perspective shift, namely the possibility of the signer to take on the role of one of the participants of the event. It includes role shift and constructed action: the former is used to report someone's speech or thought, while the latter is used to report someone's actions (for a detailed discussion on perspective shift strategies see Schlenker 2003, and Zucchi 2009 for LIS). Role shift is typically introduced by an attitude predicate (e.g. verb of saying). In this construction, indexicals tend to shift reference: a first-person pointing pronoun does not actually refer to the signer but to the participant whose role has been taken by the signer, as shown in (26).

\footnotetext{
${ }^{16}$ With the appropriate NMMs the sentence is interpreted as a standard relative clause (i.e. 'the one who completed the exam can leave').
} 
(26) Scenario: The signer saw Gianni reporting that he (Gianni) opened a door with a tool and entered the room.

$$
\text { role shift }
$$

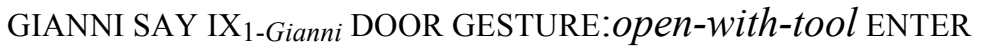

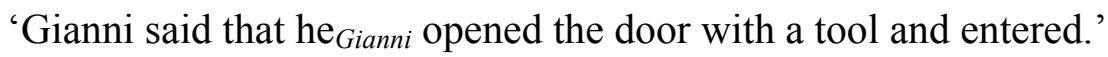

Constructed action is not introduced by any predicate: in this construction, the signer directly acts as if $s /$ he was one of the participants in the main event described by the utterance, as shown in (27).

(27) Scenario: The signer saw Gianni opening a door with a tool and entering the room. constructed action

$\mathrm{IX}_{3-\text { Gianni GESTURE:open-with-tool GESTURE:enter }}$

'Gianni opened the door with a tool and entered.'

As for role shift, agent-backgrounding readings are available only if the individual whose perspective is taken over is already referentially reduced. This is expected considering that indexical shifting is possible only if the shifted pronoun is bound by preceding linguistic material. The relevant example is shown in (28a). However, given the peculiarity of constructed action, one may expect agent backgrounding to be hard to obtain. After all, if the signer has to act as if $\mathrm{s} / \mathrm{he}$ is someone else, that someone should be at least familiar. Despite this fact, agent-backgrounding readings are easily accessible with constructed action. The relevant example is given in $(28 b)$.

role shift

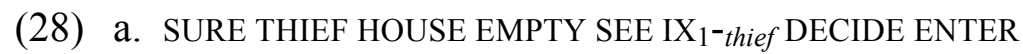

'A thief saw that my house was empty, so he thief decided to enter.'

(agent-backgrounding reading available)

constructed action

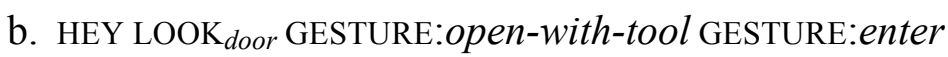

'Hey, look at the door, someone opened it with something and entered.' (agent-backgrounding reading available)

5 Impersonal vs. indefinite pronouns

Cabredo Hofherr (2008) and Barberà \& Cabredo Hofherr (2017b) provided four diagnostics to distinguish whether a certain agent-backgrounding interpretation is triggered by an indefinite marker or an impersonal one. Specifically, indefinite and impersonal constructions behave differently with respect to: i) generalizing readings, ii) corporate readings, iii) joint reference in anaphoric chains, and iv) scope ambiguity with respect to frequency adverbs. 
In this section, we investigate the semantic nature of some of the agent-backgrounding strategies used by LIS signers to see whether they involve indefinite or impersonal pronouns. For each of the criteria listed above, we tested SOMEONE, PERSON (both accompanied by ' $a b$ ' NMMs), and the null subject. Constructed actions and free relatives are complex constructions and as such they are not easily testable with these diagnostics. ${ }^{17}$

The data presented in this section show that SOMEONE and PERSON combined with ' $a b$ ' NMMs behave like indefinite pronouns, while the null subject is more similar to impersonal pronouns. For the sake of clarity, in the following examples we compare LIS data with clear cases of indefinite and impersonal constructions in Italian, namely the existential quantifier qualcuno ('someone') and the null subject.

Indefinite pronouns do not allow for generalizing readings, while impersonal pronouns do. This is shown by the Italian examples in (29). LIS data in (30) show that SOMEONE and PERSON combined with 'ab' NMMs behave like indefinite pronouns, while the null subject behaves like an impersonal pronoun.
a. In Cina qualcuno mangia gatti.
(Italian)
'In China, someone eats cats.' (not generalizing over people associated with China)
b. In Cina pro mangiano gatti.
(Italian)
'In China, people eat cats.' (generalizing over people associated with China)

(30)
$\mathrm{ab}$
a. CHINA IX ${ }_{\text {loc }}$, SOMEONE/PERSON CAT EAT
'In China, someone eats cats.' (not generalizing over people associated with China)
b. CHINA IX ${ }_{\text {LOC }}$, pro CAT EAT
'In China, people eat cats.' (generalizing over people associated with China)

Corporate readings emerge when a predicate typically select a designated group of people (e.g. raise taxes). Indefinite pronouns do not allow for corporate readings, while impersonal (null) pronouns do. Italian and LIS examples are given in (31) and (32), respectively. Again, LIS data show that SOMEONE and PERSON combined with ' $a b$ ' NMMs behave like indefinite pronouns, while the null subject example behaves like an impersonal construction.

(31) a. Qualcuno ha alzato le tasse. (Italian)

'Someone raised the taxes.' (no corporate reading)

b. pro hanno alzato le tasse. (Italian)

'The government/they raised the taxes.' (corporate reading)

$\mathrm{ab}$

(32) a. SOMEONE/PERSON TAX RAISE

'Someone raised the taxes.' (no corporate reading)

\footnotetext{
${ }^{17}$ For instance, the scope of quantifiers in sentence-final position interferes with word order. Embedding a free relative within an if-clause to obtain a donkey sentence is too complicated to be tested.
} 


\section{b. pro TAX RAISE}

'The government/they raised the taxes.' (corporate reading)

In anaphoric chains, indefinite pronouns only allow for disjoint readings, while impersonal pronouns allow for joint readings, as shown by the Italian examples in (33). LIS data are somehow intriguing in that SOMEONE with 'ab' NMMs behaves like a typical indefinite pronoun, while PERSON with 'ab' NMMs behaves similar to the null subject in an impersonal construction. The relevant pattern is shown in (34).
a. Se qualcuno ${ }_{j}$ da la risposta sbagliata, qualcuno $*_{j} / k$ viene eliminato. If someone give.3.SG the answer wrong someone get.3.SG eliminated

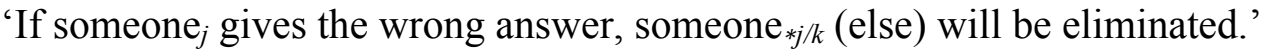
b. Se danno la risposta sbagliata, vengono eliminati.
If give.3.PL the answer wrong get.3.PL eliminated

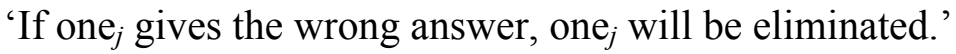

$$
\text { cond (br) }
$$

$\mathrm{ab}$

(34) a. * SOMEONE $_{j}$ ANSWER WRONG, IMMEDIATELY SOMEONE ELIMINATE $_{j}$

cond (br)

$\mathrm{ab}$

b. PERSON $_{j}$ ANSWER WRONG, IMMEDIATELY PERSON $_{j}$ ELIMINATE cond (br)

c. pro $_{j}$ ANSWER WRONG, IMMEDIATELY pro $_{j}$ ELIMINATE

'If one ${ }_{j}$ gives the wrong answer, one $e_{j}$ gets eliminated.'

However, the availability of joint reference with PERSON combined with 'ab' NMMs can be explained without assuming that it is an impersonal pronoun. Notice that the 'ab' NMMs do not spread over the second occurrence of PERSON. We claim that the absence vs. presence of the 'ab' NMMs marks the definite vs. indefinite use of PERSON. In its first occurrence, PERSON is interpreted as an indefinite DP ('a person'), while in the second occurrence it is interpreted as a definite DP ('that person'). ${ }^{18}$ Thus, a more accurate paraphrase of the example in (34b) would be if a person gives the wrong answer, that person will be eliminated. Such an interpretation is not available for SOMEONE, which is forced to be interpreted as an indefinite in both occurrences. ${ }^{19}$

Finally, indefinite pronouns show scope ambiguity with respect to clause-final frequency adverbs, while in impersonal constructions the impersonal pronoun strictly scopes below the adverb, as shown by the Italian examples in (35).

\footnotetext{
${ }^{18}$ We are extremely thankful to Jeremy Kuhn for pointing us towards this direction.

${ }^{19}$ Notice that SOMEONE may receive an indefinite interpretation even without the 'ab' facial expressions, see section 4.1.1.
} 
(35) a. Qualcuno mi ha rubato la bici due volte. (Italian)

'Someone stole my bike twice.' ( $\exists>$ twice, twice $>\exists$ )

b. Mi hanno rubato la bici due volte. (Italian)

'They stole my bike twice.' (* $\exists>$ twice, twice $>\exists$ )

Judgments on scope interactions with frequency adverbs have been extremely hard to elicit for our informants. In order to make sure that access to the relevant reading was not precluded because of other orthogonal factors (e.g. topicalized subjects are more likely to take wide scope over adverbs that are not in sentence-initial position), ${ }^{20}$ we created scenarios triggering the presence of a topicalized object (to prevent subject topicalization) and discourse stretches triggering felicitous readings. The stretches of discourse in (36) and (37) illustrate the point with the sign PERSON.

\section{(36) Intended reading: twice $>\exists$}

Scenario: I have very bad luck. I lost my bike twice.

$$
\text { top } \quad \mathrm{ab}
$$

A. IX 1 UNFORTUNATE. BIKE PERSON STEAL TWICE

'I am miserable. A person stole my bike twice.'

(Potentially ambiguous: $\exists>$ twice, twice $>\exists$ )

B. KNOW. SURE GIANNI STEAL

'I know that. I am sure that Gianni stole your bikes.'

(Addressee disambiguates: $\exists>$ twice)

A. IMPOSSIBLE. FIRST TIME YES, SECOND TIME IX 3 -Gianni PRISON

'That's impossible. The first time it could be, but the second time, he (Gianni) was in prison.' (Signer provides the intended reading: twice $>\exists$ )

(37) Intended reading: $\exists>$ twice

Scenario: I have very bad luck. I lost my bike twice.

$$
\text { top }
$$
$\mathrm{ab}$

A. IX 1 UNFORTUNATE. BIKE PERSON STEAL TWICE

'I am miserable. A person stole my bike twice.'

(Potentially ambiguous: $\exists>$ twice, twice $>\exists$ )

B. KNOW. SURE GIANNI STEAL FIRST TIME, THEN MARIA

'I know that. For sure, Gianni stole your bike and then Maria did it, too.'

(Addressee disambiguates: twice $>\exists$ )

A. IMPOSSIBLE. IX 1 KNOW MUST PERSON SAME STEAL TWICE

'It's impossible. I know that the same person must have stolen my bike twice.'

(Signer provides the intended reading: $\exists>$ twice)

\footnotetext{
${ }^{20}$ Placing the adverb in sentence-initial position would create the opposite bias. Furthermore, Cabredo Hofherr (2008) showed that scope asymmetries between impersonal and indefinite pronouns disappear if the adverb is placed in sentence-initial position.
} 
The pattern we found in LIS is given in (38). PERSON and the null subject allow for both readings, although in the case of the null subject, the reading in which the existential pronoun takes scope above the frequency adverb is less accessible according to our informants. We attribute the fact that the reading $\exists>$ twice is slightly accessible for null subjects to a possible case of accidental co-reference (i.e. the bikes got stolen twice accidentally by the same individual). Interestingly, SOMEONE only allows for a wide scope reading (i.e. it is infelicitous under the scenario (37)). With this respect, LIS SOMEONE resembles LSC ONE up (see Barberà \& Cabredo Hofherr 2017b).

(38) a. BIKE PERSON STEAL TWICE

$(\exists>$ twice, twice $>\exists)$

top

b. BIKE pro STEAL TWICE

$(\exists>$ twice, twice $>\exists)$

top

c. BIKE SOMEONE STEAL TWICE

$\left(\exists>\right.$ twice,${ }^{*}$ twice $\left.>\exists\right)$

In summary, PERSON and SOMEONE behave as indefinite pronouns, while the null subject behaves like an impersonal pronoun.

\section{Conclusions}

In this paper, we addressed how agent backgrounding is marked in LIS. The analysis of data collected from three LIS native signers through fieldwork revealed that the major semantic contributor is a particular combination of facial expressions ('ab' NMMs) co-occurring with the existential quantifier SOMEONE and the sign PERSON. Without the 'ab' facial expressions, the existential quantifier is still compatible with agent-backgrounding readings. Our study showed that agent backgrounding can also be conveyed syntactically by free relatives, perspective shift, and null subject. Finally, while SOMEONE and PERSON combined with ' $a b$ ' NMMs function as indefinite pronouns, the null subject is interpreted as an impersonal pronoun.

\section{Acknowledgments}

Many thanks are due to Mirko Santoro, Gabriele Caia, Lorenzo Laudo, Vincenza Giuranna, and Valerio Andrioli for their contribution during the elicitation sessions and to Jeremy Kuhn for valuable discussion and comments. Part of this work has been presented at SSLL2017 in Osaka, IGG 43 in Pavia, and LINGUAE seminars in Paris. We would like to thank the audience of these events for their feedback. This research received partial funding from the 
ERC Grant Agreement 324115 "FRONTSEM" (PI: Schlenker) and the Horizon 2020 project 69334 "SIGN-HUB" (PI: Carlo Cecchetto for the University of Milan-Bicocca and PI: Carlo Geraci for CNRS, IJN).

\section{References}

Barattieri, Chiara. 2006. Il periodo ipotetico in LIS. Siena: Università degli Studi di Siena dissertation.

Barberà, Gemma. 2015. The meaning of space in sign language. Reference, specificity and structure in Catalan Sign Language discourse. Berlin/Boston: De Gruyter Mouton and Ishara Press.

Barberà, Gemma \& Patricia Cabredo Hoffherr. 2017a. Backgrounded agents in Catalan Sign Language (LSC) - passives, middles or impersonals? Language 93. 767-798.

Barberà, Gemma \& Patricia Cabredo Hofherr. 2017b. Two indefinite pronouns in Catalan Sign Language (LSC). Sinn und Bedeutung 21.

Bertone, Carmela \& Anna Cardinaletti. 2011. 11 sistema pronominale della lingua dei segni italiana. In Anna Cardinaletti, Carlo Cecchetto \& Caterina Donati (eds.), Grammatica, lessico e dimensioni di variazione nella LIS, 145-160. Milano: Franco Angeli.

Branchini, Chiara. 2009. Relative libere e interrogative wh- in LIS. In Alcuni capitoli della grammatica della LIS. Atti dell'incontro di studio "La grammatica della Lingua dei Segni Italiana", 101-115. Venezia: Editrice Cafoscarina.

Branchini, Chiara \& Caterina Donati. 2009. Relatively different: Italian Sign Language relative clauses in a typological perspective. In Anikó Lipták (ed.), Correlatives crosslinguistically, 157-191. Amsterdam: John Benjamins Publishing Company.

Branchini, Chiara \& Carlo Geraci. 2011. L'ordine dei costituenti in LIS: risultati preliminari. In Grammatica, lessico e dimensioni di variazione nella LIS, 113-126. Milano: Franco Angeli.

Cabredo Hofherr, Patricia. 2008. Les pronoms impersonnels humains: syntaxe et interprétation. Modèles linguistiques vol. XXIX-1 57. 35-56.

Cecchetto, Carlo, Carlo Geraci \& Sandro Zucchi. 2006. Strategies of relativization in LIS. Natural Language and Linguistic Theory 24. 945-975.

Davidson, Kathryn \& Deanna Gagne. in prep. "More is up", pragmatic intuitions are grammaticalized in ASL as overt contextual domain restrictions. Manuscript.

Eugeni, Carlo. 2008. Una panoramica della situazione dei sordi italiani in generale e della lingua dei segni italiana in particolare. Version updated after amendments by Dino Giglioli president of the National interpreters' association (ANIMU).

Geraci, Carlo. 2012. Language policy and planning: The case of Italian Sign Language. Sign Language Studies 12(4). 494-518.

Geraci, Carlo. 2014. Spatial syntax in your hands. In Jyoti Iyer \& Leland Kusmer (eds.), NELS 44: Proceedings of the Forty-Fourth Annual Meeting of the North East Linguistic Society, vol. 1, 123-134. Amherst: GLSA.

Geraci, Carlo \& Valentina Aristodemo. 2016. An in-depth tour into sentential 
complementation in Italian Sign Language. In Roland Pfau, Markus Steinbach \& Annika Herrmann (eds.), A matter of complexity: Subordination in sign languages, 95-150. Berlin: De Gruyter.

Geraci, Carlo, Carlo Cecchetto \& Sandro Zucchi. 2008. Sentential complementation in Italian Sign Language. In Michael Grosvald \& Dionne Soares (eds.), Proceedings of the Thirtyeighth Western Conference On Linguistics, WECOL, 46-58.

von Heusinger, Klaus. 2002. Specificity and definiteness in sentence and discourse structure. Journal of Semantics 19(3). 245-274.

Liddell, Scott K. 2003. Grammar, gesture, and meaning in American Sign Language. Cambridge: Cambridge University Press.

Mantovan, Lara. 2017. Nominal modification in Italian Sign Language (LIS). Berlin: De Gruyter Mouton.

Mantovan, Lara \& Carlo Geraci. 2015. The syntax of cardinal numerals in Italian Sign Language (LIS). In Proceedings of the Forty-Fourth Annual Meeting of the North East Linguistic Society, vol. 2, 155-165.

Schlenker, Philippe. 2003. A plea for monsters. Linguistics and Philosophy 26(1). 29-120.

Schlenker, Philippe. 2011. Donkey anaphora: the view from Sign Language (ASL and LSF). Linguistics and Philosophy 34(4). 341-395.

Schlenker, Philippe. 2016. Iconic pragmatics. Manuscript available at http://ling.auf.net/lingbuzz/003215/current.pdf.

Siewierska, Anna. 2011. Overlap and complementarity in reference impersonals: Manconstructions vs. third person plural-impersonals in the languages of Europe. In Andrej Malchukov \& Anna Siewierska (eds.), Impersonal constructions. A cross-linguistic perspective, 57-90. Amsterdam/Philadelphia: John Benjamins.

Zucchi, Sandro. 2004. Monsters in the visual mode? Manuscript, Università degli Studi di Milano, http://www.filosofia.unimi.it/zucchi/NuoviFile/LISmonsters.pdf.

Zucchi, Sandro. 2009. Along the time line. Tense and time adverbs in Italian Sign Language. Natural Language Semantics 17(2). 99-139.

\section{Corresponding author's address}

Lara Mantovan

University of Milan-Bicocca; Ca' Foscari University Venice

Ca' Bembo, Dorsoduro 1075, 30123

Venice, Italy

laramantovan@hotmail.it 